\title{
The Communication Path of Film and Television Art in the Context of Micro-era
}

\author{
Sheng Huang \\ Fuzhou University of International Studies and Trade \\ Fuzhou, China 350200
}

\begin{abstract}
The Micro-era provides a variety of communication paths for film and television art works. How to select and develop these communication paths has become another problem in the development of the film industry. In order to strengthen the development of film and television art for the propagation path, this paper analyzes the origin stimulating the aesthetics progress of film and television art under the background of micro-era, and the expectation of the growth of film and television art in the context of micro-era, and finally summarizes the spread of film and television art in micro-era so as to provide a theoretical reference for the development of film and television art, and meanwhile, to provide new development directions and suggestions for expanding the path of diversified film and television art.
\end{abstract}

Keywords-micro-era; film and television art; communication path

\section{INTRODUCTION}

According to the "36th Statistical Report on Internet Development in China", the proportion of Internet users browsing in China has reached $88.9 \%$ with the supporter of smart phone, and it still shows a trend of continuous increase. The mobile internet 2017 Q2 summer report released by thirdparty data company Quest Mobile shows that the number of daily active users such as Taobao tickets and Maoyan Movie has shown an increasing trend in the movie online ticketing industry App. The online viewing ratio has continued to expand, and the number of viewings of film and television works led by Tencent Video, iQiyi, Youku and other online platforms is on the rise. The macroscopic pattern of the microera has infinitely broadened the path of film and television art. It can be considered that the spread channel of film and television art has stepped from the traditional mode to the micro-era, showing a positive development trend. How to choose the propagation path has become the developmental issue of film and television art in the micro-era. Based on this, this paper explores the propagation path of film and television art in the context of micro-era, and now the following analysis will be made.

\section{The ORIGIN Stimulating the Aesthetics}

DEVELOPMENT OF FILM AND TELEVISION ART UNDER THE BACKGROUND OF MICRO-ERA

With the advent of the micro-era, the biggest stimulus for the aesthetics of film art is that it expands the aesthetic dimension of the mass infinitely and provides another evaluation interval for the general public. When the aesthetics of film art was separated from the aesthetic limitations of classics, elites, and scales, the spread of film culture was infinitely supported and encouraged. Finally, with the help of the micro-era environment, it successfully affected the general audience's value identity for digital film and television appreciation, and expanded the aesthetic scale as well as standard of film art. In this infinitely amplified film aesthetic consciousness, its transmission path would be bound to be broadened and shape a more macroscopic cultural value definition.

\section{A. From Classics to Popularity}

The biggest feature of film and television art in the microera is decentralization, and countless film and television works are moving to the public aesthetic perspective. Although this change is related to the proliferation of online users, it is undoubtedly a positive guide to film and television art. The public evaluation, network flow, and recognition dimensions of cultural value are all dominated by the public aesthetic perspective, so the infinite enlargement of the aesthetic standards of film and television art works will appear. The following phenomena can be seen: the lines of film and television works will become popular terms; the classical plot may be widely disseminated in the self-media platform and it will be spoofed or evaluated, which is not a cultural phenomenon whose classical values can match, but a kind of universal value recognition based on the social popular culture. Therefore, after relying on the micro-era environment, the film and television art has been divorced from the restriction of the aesthetic value of the traditional classics, forming a popular orientation of the interpretation space from the perspective of the public aesthetic.

\section{B. From Elite to Grassroots}

Micro-era film and television art works are mostly digital new media products. It can be found that the film and television works are not limited to the elite production of the professional groups any more, and the grassroots actors, grassroots scripts, and grassroots directors are also qualified to occupy a place in the micro-era film and television art market. 2017-2022 China Microfilm Industry Development Prospect Analysis and Development Strategy Research Report shows that with the rapid development of online video platforms, the competition of major portals and video websites in the original 
works of film and television art has intensified. At the same time, network micro-video has also opened up a new grassroots culture. Previously, the "China Short Video Market Research Report" released by IIMedia Research showed that the scale of mobile short video users continued to expand, and the original self-media works would be expected to break through 242 million at the end of the year, with a growth rate of $58.2 \%$. The micro-era has infinitely reduced the degree of elitism of film and television art works and cleared the new grassroots path.

\section{From Scale to Cost}

In the past, the box office appeal of film and television works was more inclined to scale. When the big-cost movie occupied the leading position of the box office, the film review did not show positive recognition value feedback. In contrast, the micro-movies of rapid development in recent years, which has not invested a lot of money, have earned the good feedback from the network although their production scales have shown a trend of miniaturization, once again verifying that the micro-era film and television art is no longer limited to large-scale epic masterpieces, but rather inclined to the small cost of realism. After the video length of the film and television art is reduced to 20 minutes, it can be played, edited and photographed through the mobile intelligent terminal, and can be shared in real time on the social media platform. Film and television art materials are widely involved in micromovies, short documentary, video clips, etc., which undoubtedly saves the production cost of film and television art from various directions. When the cost is reduced and the channel is expanded, the propagation path of film and television art is surely widely extended and promoted.

\section{THE EXPECTATION OF THE DEVELOPMENT OF FILM AND TELEVISION ART COMMUNICATION UNDER THE BACKGROUND OF MICRO-ERA}

For the film and television art of the past period, the more classic the aesthetic value is; the higher the elite value of the film star is, the larger the scale is, the stronger the box office appeal will be. However, in the micro era, the inherent value of these traditional film and television art is dying one by one. The change of popular aesthetic value, the web browsing of grassroots film and television works, and the box office appeal of small-cost film works undoubtedly confirm the change of aesthetic value of film and television art in the micro era. Then, in the context of the micro-era, the development of the film and television art communication path also needs to comprehensively consider the consumption expectation to seek a new path for the progress of film and television art. Specifically, the development of film and television technology in the context of the micro-era is expected to have three characteristics: multi-media development expectation, multi-path development expectation, and multi-form development expectation.

\section{A. Multi-media Development Expectation}

The transmission path of film and television art works in the micro-era has been widely expanded, but in essence, the media still does not reach the expected saturation. Film and television art works have a higher amount of originality in large and medium-sized online media, while small online media have few original works. Although the production cost has been reduced indefinitely, the large and medium-sized network media has a higher pageview, which provides a convenient channel to select the best one in superior ones for the development of film and television art such as microfilm. This kind of channel presents a three-pronged force in the competition between iQiyi, QQ video and Youku. Unfortunately, the small network media has difficult time to obtain higher development space facing this contest. When the network resources are infinitely concentrated, the transmission path of film and television art is clearer, but in the mean time, the cultural value orientation is also prone to limitations. In fact, this kind of cultural monopoly is not conducive to the further iteration of the efficiency of film and television art communication. Therefore, the development expectation of multi-media is still the essential appeal of film and television art in the micro-era.

\section{B. Multi-path Development Expectation}

As far as the current film and television art communication path is concerned, it still does not deviate from the traditional mode of cinema production, investor operation, and third-party promotion. Although this development path is relatively stable, it is essentially less helpful for the spread of film art in the micro-era. The baptism of film and television art in the microera is to deeply explore the niche demand in the consumer market. However, if there is a lack of investment, lack of technical guidance, and lack of third-party propaganda, highquality film and television art works are still difficult to enter the consumer market. The premise and elements of maintaining the quality of works are relatively weak, and it is another shackle that restricts the development and spread of film and television art. Therefore, the expectation of multi-path development is also the communication demand that film and television art is most concerned about in the micro-era background.

\section{Multi-form Development Expectation}

The spread of film and television art requires the development of multiple forms, and such a development trend is also an inevitable result in the context of the micro-era. The film and television art products in the micro-era background are often the works of art deriving from the coordination of various creative modes. They mix a variety of cultural atmospheres, iterate a variety of cultural values, and even enrich a variety of cultural experiences. Then, their transmission path will unavoidably expand. Traditional film and television art works are usually cinema films or TV dramas broadcast by TV stations with the main creative forms of monographic films or feature films. However, in the microera background, such creative forms have undergone major changes. Micro-movies have become the cultural orientation sought by end users, and online dramas have also attracted more audiences. Then, in the transmission path of film and television art works, it is also necessary to locate the communication mechanism in the form of works in order to obtain higher word-of-mouth benefits and achieve better communication effects. 


\section{ANALYSIS OF THE COMMUNICATION PATH OF FILM AND TV ART UNDER THE BACKGROUND OF MICRO-ERA}

\section{A. Optimizing Micro-content Payment}

Under the background of the micro-era, the derivative products of film and television art works are infinitely expanded, and the placement of advertisements becomes another way to reduce costs. Although this way supports the cost iteration of film and television art works with the help of micro-platforms and it is undoubtedly an important backing for the development path of the propagation path, the lack of cultural quality behind this support can't be ignored, so the artistry of the film and television works itself is once again questioned. To broaden the communication path of film and television art in the micro-era, it's crucial to develop and optimize the payment mechanism of micro-content. For a film and television market of benign cycle, it should allow the end users to voluntarily do payment and pay for outstanding film and television works. When this healthy circular mechanism is built, film and television art works do not need to rely on advertisers to support the cost of production any more. Then the cultural value of film and television works will be deeply developed, and its publicity and reputation will first establish a communication channel that breaks through traditional values. This kind of evaluation and communication channel based on user's word-of-mouth is the development way of film and television art that needs to be developed in the micro-era. What's more, it will be a great breakthrough in the upgrading and personalization of the content quality of its film and television works.

\section{B. Supplementing Micro-cultural Sharing}

The essence of the micro-era lies in the expansion of the propagation path of film and television art works, rather than the simple scale reduction or enlargement of production. Therefore, the micro-era provides a more vivid, free, instant, and even fragmented way of communication for film and television art. Moreover, the micro-era has created a more divisive, entertaining and personalized lifestyle, allowing end users to act as an indirect cultural communication path for film and television art works. The construction of this propagation path lies in sharing. Each end user has its own communication carrier mechanism and advantages. Their WeChat moment or Microblog interaction are all potential spread genes. Taking the Douban score as an example, when the peripheral reprinting of a certain film and television work exceeds the same type of film and television works, the Douban score users will also be influenced by the citations introduced by others, so the cumulative growth rate of the evaluation scores continues to iterate. Then, if a film and television work that has been shown just now or is in the stage of being released can rely on the word-of-mouth channel maintained by the end user, it is easier for it to obtain the consumption support from this user-level, which will be further transformed into an indirect communication path. Therefore, the channels for film and television art communication should be broadly expanded, and the development and utilization of end users shall be actively explored, so that micro-culture sharing can become another positive communication path.

\section{SupportingMicro-transitional Derivation}

The development of film and television cultural art in the context of the micro-era is facing a transformation of the times. This transformation may encounter unprecedented challenges, analgesia, questioning, controversy, unaccustomedness, and inability to adapt. However, it is still impossible to deny that more film and television art communication paths are subtly affecting the film and television consumption in the terminal market. Among them, the Douban score is only a simple one, and the impact on the overall amount of film and television art is small. The new propagation path derived from the crossborder of streaming media is more irritating to end users, and the impact based on this visual communication effects is even higher. Taking the movie review function of Thunder as an example, its dissemination of film and television art culture is beyond the words, rhetoric, and articles. The function of recommending film and television works can be accompanied by the host's commentary, micro video editing, and interpretation of the film and television classic plot and so on. This kind of terminal software with the function of downloading network resources as the main function in the past period also embraces a certain propaganda function and flow currently. Then such kind of cross-border development should also be the focus of intensive development in the micro-era, and it plays an important supporting role in the comprehensive structure of film and television art in the path of cultural communication. Therefore, cross-border has become a means for film and television art to find a new communication path. New media, streaming media, and selfmedia can all serve as a positive direction for cross-border communication of film and television art.

\section{Encouraging Micro-group Comments}

In the micro era, every key user is a film critic, and each one has their own potential communication resources. If the communication resources of these end users can be used correctly, it will help the film and television art to spread rapidly in the micro era and cause social concern. The biggest feature of the micro-era is that it has a higher influence from the self-media. Relying on the self-media channel to dominate tendency of network evaluation has become an important force that many film and television art works cannot be ignored in the propaganda period. The film and television art's communication path is more concise. Mainly through microchannels such as WeChat, Weibo, Wechat Moment, Wechat Subscription, etc., the short-term evaluation can be expressed, which is more interactive and immediacy, and more targeted and independent. A small number of micro-blog opinion leaders have become the focus of influence in the dissemination of film and television art and culture. Taking the film "22" as an example, it is a long documentary about "comfort women" who survived the Japanese invasion of China produced by Sichuan Guangying Shenchu Cultural Communication Co., Ltd. and directed by Guo Ke. There are 22 "comfort women" participating in the shooting and the film is also the first "comfort women-related documentary" in China to be released with permission. The market acceptance rate of this kind of documentary is relatively low, and it is too low to match the mainstream consumption of popular entertainment. However, due to the Microblog forwarding and 
evaluation of Feng Xiaogang, it also gained a high amount of onlookers. "War Wolf II" is another example. It has enjoyed a global box office of 5.68 billion Yuan. Although it is closely related to the values advocated in the film works and production level, it is also a joint reaction caused by the active discussion and evaluation of the majority of netizens. Therefore, encouraging micro-group comments is not only a means to increase the exposure of film and television art works, but also a necessary condition for stimulating end-users' browsing. In the development trend that the importance of user experience is more attached in the micro-era, the extensive evaluation of the micro-group has become a more active communication path for film and television art. Therefore, under the background of the micro-era, the establishment of a new type of film and television art communication path does not require sharp contrast and flame war in absolute meaning. Professional and authoritative evaluations are not as good as end-users' reputation, so correspondingly, more energy should be devoted to use group comments to create positive word-ofmouth, so that a mass foundation for the spread of film and television art culture may be laid.

\section{CONCLUSION}

In summary, the micro-era has changed the creative mode of film and television art works in the past, and the development trend of multi-media, multi-path and multi-form in its communication path is more expected. Based on this, to construct the communication path of film and television art in the context of micro-era, it is necessary to optimize microcontent payment, supplement micro-culture sharing, support micro-cross-border derivation, and encourage micro-group comments. In addition, the complete construction of spread channels of film and television art in the micro-era should be further done. In the mean time, it's of great significance to expand new means and methods of communication to support the rapid development of film and television art in the microera, finally forming a more active form and space of communication, and creating the brand-new value of communication of film and television works in micro-age.

\section{REFERENCES}

[1] Liu Shuwen. On the Status Quo of Film and Television Art Creation under the Background of Micro-era[J].Art Appreciation, 2018(26): 99100. (in Chinese)

[2] Meng Lingxiao. The Current Situation of the Short Video Transmission of Video Clips in the Self-media Era: Taking Guamo "Take You to Watch X movie by X Minutes" as an Example [J].Communication Studies, 2018, 2(15): 60-61. (in Chinese)

[3] Li Yannan. Psychological Thinking of the Status of Microfilm in the Future Film and Television Market [J].Home Drama, 2017(18): 100-101.

[4] Chen Kaisi. The Trend of Film and Television Art in the Micro-era [J].Arts Criticism, 2016(11): 119-123. (in Chinese)

[5] Liu Xiaohua. Exploration and Reflection on Innovating the Industry Communication Work in "Micro-era" [J]. Technology and Enterprise, 2016 (09): 1. (in Chinese) 